activity under natural conditions or all oil in reservoirs would be extensively biodegraded.

The new observations ${ }^{3}$ do not completely rule out the presence of indigenous bacterial populations, and it would be interesting to know whether there is a correlation between the start and duration of seawater injection and increases in numbers of bacteria emerging with production fluids. Also it would be important to be able to confirm the activity of bacteria under in situ oil reservoir conditions, and to determine their contribution to sulphide production compared to that of geochemical processes ${ }^{1}$. The results presented do, however, provide firm evidence for the presence of a subterranean biosphere in oil reservoirs; moreover they are consistent with demonstrations of the existence of other deep biospheres in aquifers $^{12}$ and marine sediments ${ }^{13}$, which together indicate that the biosphere is not just a thin veneer on the geosphere. They are bound to fuel the debate on the existence of a hot, subterranean biosphere $^{14}$.

John Parkes is in the Department of Geology, and James Maxwell in the Department of Chemistry, University of Bristol, Bristol BS8 1RI, UK.

\title{
The silicon chameleon
}

\section{Leigh Canham}

GooD news for those working on applications of light-emitting diodes for complex displays: the colour of light emitted by a porous silicon diode can vary appreciably depending on the applied voltage, say $\mathrm{A}$. Bsiesy et al. ${ }^{1}$.

Porous silicon has excited widespread interest since 1990, when its striking lightemitting properties were first reported and ascribed to quantum size effects ${ }^{2}$. It is generally created by using hydrofluoric acid to etch an array of holes ('pores') electrochemically into crystalline silicon wafers. Electron microscopy soon revealed the resulting columnar microstructure $^{3}$ of luminescent material. The skeleton left behind retains the substrate crystallinity and can have very low dimensionality, as the pores typically have widths of 2--20 nanometres and are closely packed together. Structures of high porosity (void fraction) are capable of delivering very efficient (1-10 per cent) visible photoluminescence; this is on a par with well-established luminescent semiconductors such as GaAs. For applications involving light-emitting diodes (LEDs), however, efficient electroluminescence has to be achieved. In 1992, this was shown by two independent groups ${ }^{4,5}$ to be feasible, and the LED structure used was the one studied by Bsiesy and colleagues. It is remarkable for a number of reasons.

To start with, at first sight it is not clear why it should work at all. In this device, a liquid electrolytic contact containing a specific redox species (the persulphate $\mathrm{S}_{2} \mathrm{O}_{8}^{2-}$ ion) infiltrates the porous layer on the bulk silicon substrate. When a cathodic (negative) voltage is applied to the silicon with respect to a metallic anode (platinum or other metal), visible electroluminescence is obtained from layers that produce visible photoluminescence. But such porous structures in air are known to be highly resistive. So when we fill the pores with a conductive liquid, why doesn't virtually all current flow through that liquid into the bulk silicon, effectively short-circuiting the resistive porous silicon? Indeed, that is precisely what happens when the layer is being formed in the etchant.

Second, as Bsiesy and colleagues show, a single layer can produce any of four colours depending on excitation conditions. The range of spectral tunability is much greater than seen previously ${ }^{5}$. For their layers, fabricated under illumination, a shift of electroluminescence as large as $270 \mathrm{~nm}$ is obtained for an external bias variation of only $0.6 \mathrm{~V}$. This compares extremely favourably with tunable colour devices using stacked zinc sulphide phosphor structures without filters, where red-green colour variation requires a 30 $\mathrm{V}$ change in bias ${ }^{6}$. Third, it is remarkable that the electroluminescence output band can be spectrally so much narrower (its full width at half maximum is about 0.25 $\mathrm{eV}$ ) than the photoluminescent band of the same layer (about $0.6 \mathrm{eV}$ ).

The first quandary is explicable if the electrolyte does two things. Not only do its persulphate ions efficiently inject holes into the silicon skeleton, but under cathodic bias its cations (such as $\mathrm{Na}^{+}$and $\mathrm{H}^{+}$) dope that skeleton and thereby dramatically lower its resistivity. They do this by charging the surfaces of the silicon columns. In much the same way that electrolytes can induce very high electron accumulation at the surface of bulk silicon ${ }^{7}$, a one-dimensional electron gas is generated in the interconnected columns of porous silicon. Efficient electroluminescence at low biases can then result from recombination of localized holes ("minority carriers') with delocalized electrons ('majority carriers') that flow along the wires.

The second puzzle can be addressed within the framework of quantum- confinement effects. A key prediction of such models is that light emission wavelength is a function of size. The narrower the 'quantum wire' or 'quantum dot', the larger the bandgap and the shorter the wavelength of emission. Theoretical calculations suggest that a 30 - $\AA$ bare silicon quantum wire should emit deep red light. Native oxide growth can reduce the silicon core to about $20 \AA$ and result in orange emission. Even narrower structures would then correspond to yellow and green emission, the band gap being very sensitive to wire size and shape in this regime. Porous silicon layers are inhomogeneous in the sense that they generally contain a range of wire widths ('crystallite sizes'). Bsiesy et al. ${ }^{1}$ suggest that this diode structure, where contact is made to the whole of the nanostructure, is capable of selective excitation - spectral tunability is controlled by the cathodic bias which enables smaller and smaller crystallites to be excited. Hence the dramatic blue-shift with increasing bias.

That brings us to the third puzzle. One important issue not addressed by Bsiesy et $a l$. is the role of vertical inhomogeneity in the layer. Many luminescent structures show strong evidence for porosity gradients with depth ${ }^{8}$. This is a consequence of their formation mechanism: the top of the layer, having been in the etching solution longer than the bottom, has slightly wider pores. The silicon skeleton is then smaller at the top of the layer, resulting in shorter-wavelength emission. If this effect is important, the red emission could predominantly arise from the bottom of their layer, the green from the top. Then the increasing cathodic bias, by rendering more and more of the layer conductive, would generate a blueshift. How sensitive, I wonder, was the photoluminescence to excitation wavelength and intensity?

Scientists striving to make use of semiconductor nanostructures in novel devices generally apply lithographic techniques. Beautifully regular arrays of structures with narrow size distributions are usually used as a yardstick of the success of the fabrication process. So it is ironic that here we have a nanostructure in which, as Bsiesy et al. ${ }^{1}$ suggest, the very inhomogeneity produces the desirable characteristic of tunable colour emission.

Leigh Canham is at the Defence Research Agency, St Andrews Road, Great Malvern WR14 3PS, UK

\footnotetext{
1. Bsiesy, A. et al. Phys. Rev. Lett. 71.637-640 (1993).

Canham. L. T Appl. Phys Lett. 57, 1046 (1990)

3. Cullis, A. G. \& Canham, L. T. Nature 353, 335-338 (1991).

4. Bressers, P. M. M. C. et al. Appl. Phys. Lett. 61, 108 (1992).

5. Canham, L. T. et al. Appl. Phys. Lett. 61, 2563(1992)

6 Fukao, R et al IEEE Trans. Electr. Dev 40,64 (1993).

7. Tardella A. \& Chazalviel. J N Phys. Rev. B32. 2439 (1985).

8. inoue, K. etal. Jpn Appl. Phys. 31, L997 (1992)
} 